\title{
SOME ANALOGUES OF MARKOV AND DESCARTES SYSTEMS FOR RIGHT DISFOCALITY
}

\author{
P. W. ELOE AND JOHNNY HENDERSON
}

\begin{abstract}
A necessary and sufficient condition for the disconjagacy of the $n$th order linear differential equation $y^{(n)}+a_{1}(x) y^{(n-1)}+\cdots+a_{n}(x) y=0$ on a compact interval $I$ is that there exists a system of solutions $y_{1}, \ldots, y_{n}$ such that any one of the following is satisfied: (i) $W\left(y_{1}, \ldots, y_{k}\right)>0,1 \leq k \leq n$, on $I$; (ii) $W\left(y_{i_{1}}, \ldots, y_{i_{k}}\right)>0,1 \leq i_{1}<\cdots<i_{k} \leq n, 1 \leq k \leq n$, on $I$; or (iii) $W\left(y_{i}, y_{i+1}, \ldots, y_{i+k-1}\right)>0,1 \leq i \leq n-k+1,1 \leq k \leq n$, on $I$.

Necessary and sufficient criteria for the right disfocality of the linear differential equation on the compact interval $I$ are established in terms of systems of solutions satisfying conditions which are analogous to those given in (i), (ii), (iii).
\end{abstract}

1. Introduction. We consider the $n$th order linear differential equation

$$
y^{(n)}+a_{1}(x) y^{(n-1)}+\cdots+a_{n}(x) y=0,
$$

where each $a_{i}$ is a continuous real-valued function defined on an open interval 0 . Let $I=[a, b]$ be a compact subinterval of 0 . If the only solution of (1) having at least $n$ zeros on $I$ is the zero solution, then (1) is said to be disconjugate on $I$. If the only solution of (1) satisfying $y^{(i-1)}\left(x_{i}\right)=0$ for some $x_{i} \in I, 1 \leq i \leq n, x_{1} \leq \cdots \leq x_{n}$, is the zero solution, then (1) is said to be right disfocal on $I$. Note that by Rolle's Theorem, the right disfocality of (1) on $I$ implies the disconjugacy of (1) on $I$. In this paper, we will establish necessary and sufficient criteria for the right disfocality of (1) on $I$ which are analogous to well-known necessary and sufficient criteria for the disconjugacy of $(1)$ on $I$.

If $y_{1}, \ldots, y_{n} \in C^{n}(I)$ satisfy

$$
W\left(y_{1}, \ldots, y_{k}\right)>0, \quad 1 \leq k \leq n,
$$

on $I$ where $W$ denotes the Wronskian determinant, then the functions $y_{1}, \ldots, y_{n}$ are said to form a Markov system on $I$. The functions $y_{1}, \ldots, y_{n}$ are said to form a Descartes system on $I$ if

$$
W\left(y_{i_{1}}, \ldots, y_{i_{k}}\right)>0, \quad 1 \leq i_{1}<\cdots<i_{k} \leq n, 1 \leq k \leq n,
$$

on $I$. Furthermore, we shall say that $y_{1}, \ldots, y_{n}$ form a Fekete system on $I$ if

$$
W\left(y_{i}, y_{i+1}, \ldots, y_{i+k-1}\right)>0, \quad 1 \leq i \leq n-k+1,1 \leq k \leq n,
$$

on $I$.

Pólya [6] showed that (1) is disconjugate on $I$ if and only if (1) has a Markov system of solutions on I. In Coppel [2], it is shown that (1) has a Markov system of

Received by the editors August 30, 1984 and, in revised form, January 30, 1986.

1980 Mathematics Subject Classification (1985 Revision). Primary 34B05, 34B10, 34C10.

Key words and phrases. Disconjugate, right disfocal, Markov system, Descartes system, Fekete system. 
solutions on $I$ if and only if (1) has a Descartes system of solutions on $I$; moreover, it is also shown in [2] that any Fekete system of functions is also a Descartes system of functions. Thus, the existence of either a Markov, Fekete, or Descartes system of solutions of (1) on $I$ is equivalent to the disconjugacy of (1) on $I$.

In this paper, we consider systems of solutions of (1), analogous to the above Markov, Fekete, and Descartes systems, whose existence are equivalent to the right disfocality of (1) on $I$. In that direction, Muldowney [4] obtained the following result:

THEOREM 1.1. The equation (1) is right disfocal on I if and only if there exist solutions $y_{1}, \ldots, y_{n}$ of (1) such that

$$
W\left(y_{1}^{(j-1)}, \ldots, y_{k}^{(j-1)}\right)>0, \quad 1 \leq j \leq n-k+1,1 \leq k \leq n,
$$

on $I$.

The system of solutions of (1) considered in Muldowney's result is analogous in some sense to a Fekete system of solutions. Later in a much more extensive paper, Muldowney [5] extended this type of criteria to a general class of boundary value problems for (1) which included both the conjugate and the right focal types of problems. Although part of the results of this paper can be viewed as special cases of some of those of [5], we establish herein criteria solely for the right disfocality of (1) in terms of systems of solution of (1) which are in very close analogy to Markov and Descartes systems of solutions.

2. Definitions and determinant identities. In this section, we will define systems of functions analogous to those considered above, and we will prove a useful determinant identity.

Definitions. (i) Let $y_{1}, \ldots, y_{n} \in C^{n}(I)$. For $1 \leq k \leq n$ and indices $1 \leq i_{1}$ $<\cdots<i_{k} \leq n$, define

$$
D^{k}\left(y^{i_{1}}, \ldots, y^{i_{k}}\right) \equiv \operatorname{det}\left[y_{l}^{\left(i_{j}-1\right)}(x)\right]_{1 \leq l, j \leq k},
$$

$x \in I$. If

$$
D^{k}\left(y^{n-k+1}, y^{n-k+2}, \ldots, y^{n}\right)>0, \quad 1 \leq k \leq n,
$$

on $I$, we shall say that $y_{1}, \ldots, y_{n}$ form a $D$-Markov system on $I$. The functions $y_{1}, \ldots, y_{n}$ will be said to form a $D$-Descartes system on $I$ if

$$
D^{k}\left(y^{i_{1}}, \ldots, y^{i_{k}}\right)>0, \quad 1 \leq i_{1}<\cdots<i_{k} \leq n, 1 \leq k \leq n,
$$

on $I$. Finally, if

$$
D^{k}\left(y^{i}, y^{i+1}, \ldots, y^{i+k-1}\right)>0, \quad 1 \leq i \leq n-k+1,1 \leq k \leq n,
$$

on $I$, we shall say that $y_{1}, \ldots, y_{n}$ form a $D$-Fekete system on $I$.

(ii) Let $A=\left[a_{i j}\right]_{1 \leq i, j \leq n}$ be a real $n \times n$ matrix. For $1 \leq k \leq n$ and indices $1 \leq i_{1}<\cdots<i_{k} \leq n$, define

$$
D^{k}\left(a^{i_{1}}, a^{i_{2}}, \ldots, a^{i_{k}}\right) \equiv \operatorname{det}\left[a_{i_{j} l}\right]_{1 \leq j, l \leq k},
$$

and for $\beta=\left(b_{1}, \ldots, b_{k}\right) \in R^{k}$ and $1 \leq j \leq k$, define

$$
D_{j}^{k}\left(a^{i_{1}}, \ldots, \beta, \ldots, a^{i_{k-1}}\right) \equiv D^{k}\left(\alpha^{1}, \ldots, \alpha^{k}\right)
$$


where

$$
\begin{array}{rlrl}
\alpha^{l} & =a^{i_{l}}, & & 1 \leq l \leq j-1, \\
\alpha^{j} & =\beta, & \\
\alpha^{l}=a^{i_{l-1}}, & & j+1 \leq l \leq k .
\end{array}
$$

The following determinant identity is an application of Sylvester's identity $[\mathbf{3}]$. Muldowney [4, Lemma 2] has established this result for the case $j=2$; the cases $2<$ $j \leq k$ follow from [4] by elementary row operations and properties of determinants.

LEMMA 2.1. Let $2 \leq k \leq n$, indices $1 \leq i_{1}<\cdots<i_{k} \leq n$, and $\beta \in R^{k}$ be given. Then for each $2 \leq j \leq k$,

$$
\begin{aligned}
D_{j-1}^{k-1}( & \left(a^{i_{2}}, \ldots, \beta, \ldots, a^{i_{k-1}}\right) D^{k}\left(a^{i_{1}}, \ldots, a^{i_{k}}\right) \\
= & D^{k-1}\left(a^{i_{1}}, \ldots, a^{i_{k-1}}\right) D_{j-1}^{k}\left(a^{i_{2}}, \ldots, \beta, \ldots, a^{i_{k}}\right) \\
& +D^{k-1}\left(a^{i_{2}}, \ldots, a^{i_{k}}\right) D_{j}^{k}\left(a^{i_{1}}, \ldots, \beta, \ldots, a^{i_{k-1}}\right) .
\end{aligned}
$$

COROLLARY 2.2. Let $1 \leq h \leq n$ be given. If $D^{m}\left(a^{i}, a^{i+1}, \ldots, a^{i+m-1}\right)>0$, $1 \leq i \leq n-m+1,1 \leq m \leq h$, then $D^{k}\left(a^{i_{1}}, \ldots, a^{i_{k}}\right)>0$ for all sets of indices satisfying $1 \leq i_{1}<\cdots<i_{k} \leq n, 1 \leq k \leq h$.

PROOF. The proof is by induction on $k$ and the difference $i_{k}-i_{1}$. For $k=1$, the assertion is true. Now let $1<k \leq h$ and assume $D^{l}\left(a^{i_{1}}, \ldots, a^{i_{l}}\right)>0$ for all sets of indices satisfying $1 \leq i_{1}<\cdots<i_{l} \leq n, 1 \leq l<k$.

Note that from the hypotheses of the corollary, $D^{k}\left(a^{i_{1}}, \ldots, a^{i_{k}}\right)>0$ for all sets of indices satisfying $1 \leq i_{1}<\cdots<i_{k} \leq n, i_{k}-i_{1}=k-1$. As our second assumption, let $\alpha>k-1$ and assume $D^{k}\left(a^{i_{1}}, \ldots, a^{i_{k}}\right)>0$ for all sets of indices satisfying $1 \leq i_{1}<\cdots<i_{k} \leq n, k-1 \leq i_{k}-i_{1}<\alpha$.

Now let $1 \leq i_{1}<\cdots<i_{k} \leq n$ with $i_{k}-i_{1}=\alpha$ be given. Then $i_{j}-i_{j-1}>1$ for some $2 \leq j \leq k$. Set $\beta=\left(b_{1}, \ldots, b_{k}\right)=\left(a_{i_{j-1}+1,1}, \ldots, a_{i_{j-1}+1, k}\right)$. Then by the induction hypotheses on $k, D_{j-1}^{k-1}\left(a^{i_{2}}, \ldots, \beta, \ldots, a^{i_{k-1}}\right), D^{k-1}\left(a^{i_{1}}, \ldots, a^{i_{k-1}}\right)$, and $D^{k-1}\left(a^{i_{2}}, \ldots, a^{i_{k}}\right)$ are all positive. Further, by the induction hypotheses on $i_{k}-i_{1}$, $D_{j-1}^{k}\left(a^{i_{2}}, \ldots, \beta, \ldots, a^{i_{k}}\right)$ and $D_{j}^{k}\left(a^{i_{1}}, \ldots, \beta, \ldots, a^{i_{k-1}}\right)$ are both positive. Applying Lemma 2.1, we have that $D^{k}\left(a^{i_{1}}, \ldots, a^{i_{k}}\right)>0$.

We observe here that if $y_{1}, \ldots, y_{n} \in C^{n}(I)$ form a $D$-Fekete system, then as an application of Corollary $2.2, y_{1}, \ldots, y_{n}$ form a $D$-Descartes system. Such an application of the corollary can be viewed as a special case of a result of Muldowney's [5, Proposition 1].

3. Criteria for right disfocality. In this section, we establish a number of conditions which are equivalent to the right disfocality of (1) on the compact interval $I=[a, b]$.

DEFINITION. We shall say that (1) is two-point right disfocal on $I$ if each boundary value problem for (1) satisfying the conditions

$$
\begin{array}{ll}
y^{(i-1)}\left(x_{1}\right)=0, & 1 \leq i \leq k, \\
y^{(i-1)}\left(x_{2}\right)=0, & k+1 \leq i \leq n,
\end{array}
$$

$a \leq x_{1}<x_{2} \leq b, 1 \leq k \leq n-1$, has only the trivial solution.

The first result is due to Muldowney [4, Proposition 1]. 
THEOREM 3.1. The equation (1) is two-point right disfocal on $I$ if and only if (1) is right disfocal on $I$.

The next theorem relates the existence of a $D$-Markov system on a compact interval $[c, d]$ to the existence of a $D$-Fekete system on the half-open interval $(c, d]$.

THEOREM 3.2. Let $[c, d]$ be a compact subinterval of the reals. Suppose that $y_{1}, \ldots, y_{n}$ form a D-Markov system on $[c, d]$ such that $y_{1}(c)=y_{1}^{\prime}(c)=\cdots=$ $y_{1}^{(n-2)}(c)=0$. Then $y_{1}, \ldots, y_{n}$ form a $D$-Fekete system on $(c, d]$.

PROOF. $y_{1}(x)$ satisfies $D^{1}\left(y^{n}\right)=y_{1}^{(n-1)}(x)>0$ on $[c, d]$, as well as the conditions $y_{1}(c)=y_{1}^{\prime}(c)=\cdots=y_{1}^{(n-1)}(c)=0$; hence, $D^{1}\left(y^{i}\right)=y_{1}^{(i-1)}(x)>0$ on $(c, d]$, $1 \leq i \leq n$. The proof now proceeds by a double induction. Let $k>1$ and assume for $1 \leq l<k$ that $D^{l}\left(y^{i}, y^{i+1}, \ldots, y^{i+l-1}\right)>0$ on $(c, d], 1 \leq i \leq n-l+1$. Note, from the hypotheses of the theorem, that $D^{k}\left(y^{n-k+1}, \ldots, y^{n}\right)>0$ on $[c, d]$, and so, let $i<n-k+1$ and assume for $i<h \leq n-k+1$ that $D^{k}\left(y^{h}, \ldots, y^{h+k-1}\right)>0$ on $(c, d]$.

Now consider $D^{k}\left(y^{i}, \ldots, y^{i+k-1}\right)$. Noting that

$$
\frac{d}{d x} D^{k}\left(y^{i}, \ldots, y^{i+k-1}\right)=D^{k}\left(y^{i}, \ldots, y^{i+k-2}, y^{i+k}\right)
$$

and applying Lemma 2.1 to $D^{k}\left(y^{i}, \ldots, y^{i+k-2}, y^{i+k}\right)$, with $j=k$ and

$$
\beta=\left(y_{1}^{(i+k-2)}(x), \ldots, y_{k}^{(i+k-2)}(x)\right)
$$

we have

$$
\begin{aligned}
D^{k-1} & \left(y^{i+1}, \ldots, y^{i+k-1}\right) D^{k}\left(y^{i}, \ldots, y^{i+k-2}, y^{i+k}\right) \\
& =D^{k-1}\left(y^{i}, \ldots, y^{i+k-2}\right) D^{k}\left(y^{i+1}, \ldots, y^{i+k}\right) \\
& +D^{k-1}\left(y^{i+1}, \ldots, y^{i+k-2}, y^{i+k}\right) D^{k}\left(y^{i}, \ldots, y^{i+k-1}\right) .
\end{aligned}
$$

Set

$$
\begin{gathered}
f(x)=D^{k}\left(y^{i}, \ldots, y^{i+k-1}\right), \quad g(x)=D^{k-1}\left(y^{i+1}, \ldots, y^{i+k-1}\right), \\
p(x)=D^{k-1}\left(y^{i}, \ldots, y^{i+k-2}\right) D^{k}\left(y^{i+1}, \ldots, y^{i+k}\right) \\
q(x)=D^{k-1}\left(y^{i+1}, \ldots, y^{i+k-2}, y^{i+k}\right) .
\end{gathered}
$$

Then $f(x)=D^{k}\left(y^{i}, \ldots, y^{i+k-1}\right)$ satisfies the initial value problem

$$
g(x) f^{\prime}(x)=p(x)+q(x) f(x), \quad f(c)=0 .
$$

Note that the induction hypotheses on $k$ imply $g(x)>0$ on $(c, d]$, that the induction hypotheses on both $k$ and $i$ imply $p(x)>0$ on $(c, d]$, and that the hypotheses on $k$ coupled with Corollary 2.2 imply $q(x)>0$ on $(c, d]$. We further note that, since $d g(x) / d x=q(x), g(x)$ is strictly increasing on $[c, d]$.

Now it is also the case that $g(c)=0$; thus, given $\delta>0$ and $c+\delta<x \leq d$, it follows from the differential equation satisfied by $f(x)$ that

$$
f(x)=g(x) \int_{c+\delta}^{x} \frac{p(t)}{g^{2}(t)} d t+\frac{g(x)}{g(c+\delta)} f(c+\delta) .
$$


As a consequence of the preceding paragraph, it follows that $f(x)>f(c+\delta)$ for all $x>c+\delta$. Taking limits and invoking the initial condition $f(c)=0$, it follows in turn that

$$
f(x)=D^{k}\left(y^{i}, \ldots, y^{i+k-1}\right)>0 \text { on }(c, d] .
$$

Therefore, by induction, the family $y_{1}, \ldots, y_{n}$ forms a $D$-Fekete system on $(c, d]$.

We now present the main result of the paper.

THEOREM 3.3. The following are equivalent:

(i) Equation (1) is right disfocal on $I$.

(ii) Equation (1) is two-point right disfocal on $I$.

(iii) Equation (1) has a D-Markov system of solutions $y_{1}, \ldots, y_{n}$ on $I$ which satisfy the partial set of initial conditions,

$$
\begin{aligned}
& y_{i}^{(j-1)}(a)=0, \quad 1 \leq j \leq n-i, \\
& (-1)^{i-1} y_{i}^{(n-i)}(a)>0, \quad 1 \leq i \leq n .
\end{aligned}
$$

(iv) Equation (1) has a D-Markov system of solutions $y_{1}, \ldots, y_{n}$ on I such that $y_{1}$ satisfies $y_{1}^{(i-1)}(a)=0,1<i<n-1$.

(v) Equation (1) has D-Fekete system of solutions on $I$.

(vi) Equation (1) has a D-Descartes system of solutions on $I$.

PROOF. The pattern of proof will be (i) implies (ii) implies (iii) implies (iv) implies (v) implies (i). The equivalence of (v) and (vi) is immediate from the definitions and Corollary 2.2 .

That (i) implies (ii), and in fact the equivalence of the two statements, follows from Theorem 3.1. For (ii) implies (iii), let us assume that (1) is two-point right disfocal on $I$ and let $y_{1}, \ldots, y_{n}$ be the fundamental set of solutions of (1) satisfying the initial conditions

$$
\begin{aligned}
& y_{i}^{(j-1)}(a)=0, \quad 1 \leq j \leq n, j \neq n-i, \\
& y_{i}^{(n-i)}(a)=(-1)^{i-1}, \quad 1 \leq i \leq n .
\end{aligned}
$$

We observe that $D^{k}\left(y^{n-k+1}, \ldots, y^{n}\right)>0$ for $x=a, 1 \leq k \leq n$. If

$$
D^{l}\left(y^{n-l+1}, \ldots, y^{n}\right)=0,
$$

for some $1 \leq l \leq n$ and some $x=\tau \in(a, b]$, then there exists a nontrivial solution $z=c_{1} y_{1}+\cdots+c_{l} y_{l}$ of (1) such that

$$
z^{(n-l)}(\tau)=z^{(n-l+1)}(\tau)=\cdots=z^{(n-1)}(\tau)=0 .
$$

By construction, we also have

$$
z(a)=z^{\prime}(a)=\cdots=z^{(n-l-1)}(a)=0 .
$$

The existence of such a solution $z$ is a contradiction to the two-point right disfocality of (1), and we conclude that $D^{k}\left(y^{n-k+1}, \ldots, y^{n}\right)>0,1 \leq k \leq n$, on $[a, b]$; thus, (iii) is satisfied.

The fact that (iii) implies (iv) is trivially true. In proving that (iv) implies (v), let us assume that $y_{1}, \ldots, y_{n}$ form a $D$-Markov system of solutions of (1) on $I$ with $y_{1}^{(i-1)}(a)=0,1 \leq i \leq n-1$. It follows from the continuous dependence of the determinants $D^{k}$ on solutions of (1) and from the continuous dependence of 
solutions of (1) on initial conditions that, for $\tau<a$ and sufficiently near to $a$, if $u$ is the solution of (1) satisfying the initial conditions

$$
u^{(i-1)}(\tau)=y_{1}^{(i-1)}(a), \quad 1 \leq i \leq n,
$$

then $u, y_{2}, \ldots, y_{n}$ form a $D$-Markov system of solutions of $(1)$ on the interval $[\tau, b]$. As a consequence of Theorem $3.2, u, y_{2}, \ldots, y_{n}$ form a $D$-Fekete system of solutions of $(1)$ on $(\tau, b]$, hence a $D$-Fekete system of solutions on $[a, b]$. Therefore, $(\mathrm{v})$ holds.

For (v) implies (i), we cite Muldowney's result which we stated in the Introduction as Theorem 1.1, and the proof is complete.

REMARKS. (a) Concerning the proof of Theorem 3.3, we remark further that various results in Muldowney's paper [5, Proposition 1 and Theorem 1] are relevant to some of the implications.

(b) That (1) has a $D$-Markov system of solutions on $I$ is not sufficient for the right disfocality of $(1)$ on $I$. For example, $y^{\prime \prime}+y=0$ is not right disfocal on $I=[a, b]$ if $b-a \geq \pi / 2$. However, the solutions $y_{1}(x)=-\cos x, y_{2}(x)=-\sin x$ form a $D$-Markov system of solutions on $(0, \pi)$, and thus, form a $D$-Markov system of solutions on compact subintervals of $(0, \pi)$ of length greater than $\pi / 2$.

(c) Markov and Descartes systems play an important role in approximation theory; see, for example Cheney [1]. Our definitions used here are slight modifications of those usual in approximation theory.

\section{REFERENCES}

1. E. W. Cheney, Introduction to approximation theory, McGraw-Hill, New York, 1966.

2. W. Coppel, Disconjugacy, Lecture Notes in Math., vol. 220, Springer-Verlag, New York and Berlin, 1971.

3. F. R. Gantmacher, The theory of matrices, vol. I, Chelsea, New York, 1960.

4. J. S. Muldowney, A necessary and sufficient condition for disfocality, Proc. Amer. Math. Soc. 74 (1979), 49-55.

5. __ On invertibility of linear ordinary differential boundary value problems, SIAM J. Math. Anal. 12 (1981), 368-384.

6. G. Pólya, On the mean-value theorem corresponding to a given linear homogeneous differential equation, Trans. Amer. Math. Soc. 24 (1922), 312-324.

Department of Mathematics, University of Dayton, Dayton, Ohio 45469

Department of Mathematics, Auburn University, Auburn, Alabama 36849 